\title{
COLOURS: A SCIENTIFIC APPROACH
}

Venkatesh Bharadwaj

Former Professor, Soil Science and Head, Agrometeorology Department, G.B.Pant University of Agriculture and Technology, Pantnagar, 263145, U.S.Nagar (Uttarakhand), India.

Email:venkatbh@hotmail.com

Colour is visual identification or perception of a property derived from spectrum of light by human eyes. When a beam of light from sun or light source passes through a glass prism, it dispersed in to seven colours of rainbow. Sunlight has a high color temperature, a fairly uniform spectrum and is considered a standard for white light.

A rainbow is an optical and meteorological phenomenon caused by reflection and refraction of light in water droplets present in the atmosphere. The rainbow colours are violet, indigo, blue, green, yellow, orange and red (vibgyor). Electromagnetic radiation in the visible range is generally called as light.
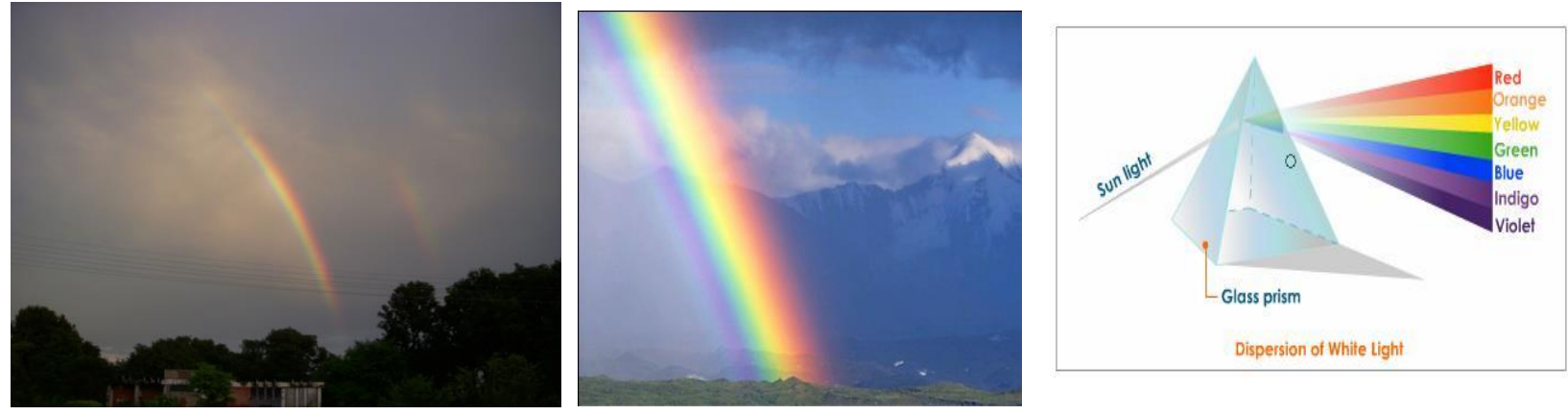

Color are also associated with objects or materials and based on their optical properties such as light absorption, reflection or emission.
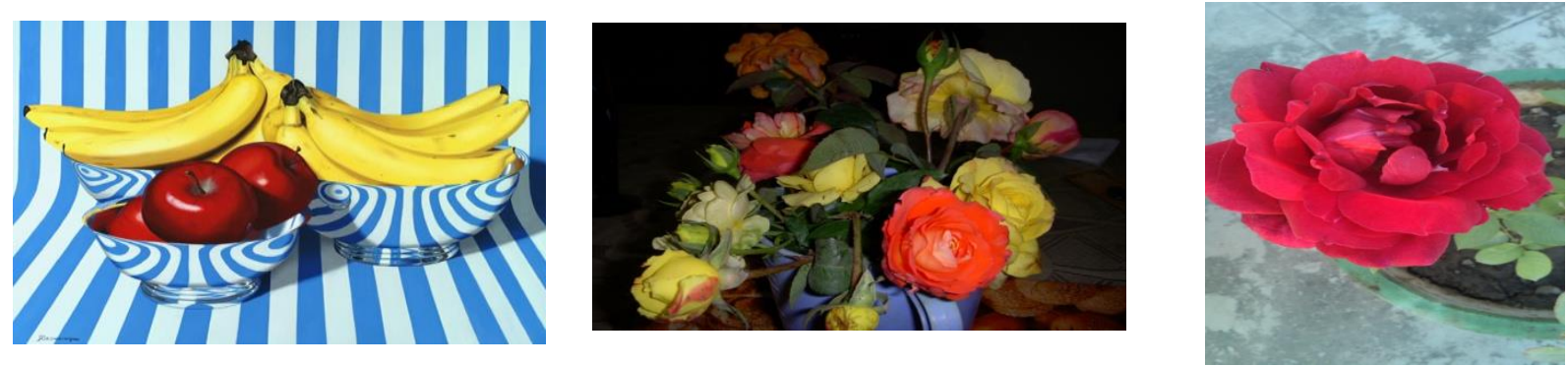

2. Color science - Science of color study is called as Colorimetry, Chromatics or simply Color science. It includes the perception of color by the human eye and brain, the origin of color in materials, color theory in art and the physics of electromagnetic radiation (EMR) in the visible range i.e. light. Colors can be identified numerically by their wave lengths i.e.635 to 590 $\mathrm{nm}$ (yellow colour), by their coordinates as $\mathbf{5 P} \mathbf{5 / 1 0}$ as well as by their numerical values in Roman like, I light green, II yellow, III red, IV blue, V dark green, VI orange, VII grey, VIII violet etc. 


\section{INTERNATIONAL JOURNAL Of RESEARCH -GRANTHAALAYAH \\ A knowledge Repository}

Art

The colours of the visible light

\begin{tabular}{|l|l|l|}
\hline colour & $\begin{array}{l}\text { wavelength } \\
\text { interval }\end{array}$ & $\begin{array}{l}\text { frequency } \\
\text { interval }\end{array}$ \\
\hline$\underline{\text { red }}$ & $\sim 700-635 \mathrm{~nm}$ & $\sim 430-480 \mathrm{THz}$ \\
\hline orange & $\sim 635-590 \mathrm{~nm}$ & $\sim 480-510 \mathrm{THz}$ \\
\hline yellow & $\sim 590-560 \mathrm{~nm}$ & $\sim 510-540 \mathrm{THz}$ \\
\hline green & $\sim 560-490 \mathrm{~nm}$ & $\sim 540-610 \mathrm{THz}$ \\
\hline$\underline{\text { blue }}$ & $\sim 490-450 \mathrm{~nm}$ & $\sim 610-670 \mathrm{THz}$ \\
\hline violet & $\sim 450-400 \mathrm{~nm}$ & $\sim 670-750 \mathrm{THz}$ \\
\hline
\end{tabular}

$5 P$

$5 / 10$

3. Electromagnetic spectrum - Most light sources are mixtures of various wavelengths of light and different light sources emit light at numerous wavelengths. But human eye can perceive wave lengths in the range of 400 (390) to $700 \mathrm{~nm}$ only. This is known as visible light or visible EMR.

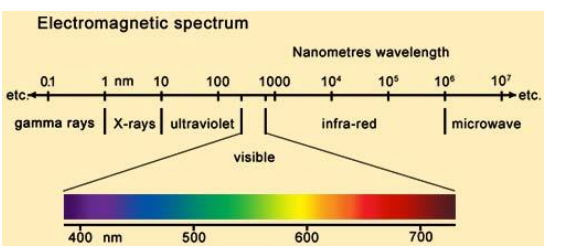

Likewise green plants use 400 to $700 \mathrm{~nm}$ wavelength of EMR for photosynthesis. This is called as photo synthetically active radiation (PAR). Photosynthesis makes food of plants.

4. Colour bands - Everywhere people identify colors in the same way. However, the names are different according to their language. A common list includes six main bands: red, orange, yellow, green, blue, and violet. A seventh color between blue and violet is indigo. Blue is nearer to cyan and indigo is similar to dark blue indigo dye. The human eye can distinguish about One Crore different colors. 


\section{INTERNATIONAL JOURNAL Of RESEARCH -GRANTHAALAYAH

Mixing of green light $(530 \mathrm{~nm})$ and blue light $(460 \mathrm{~nm})$ produces cyan light $(485 \mathrm{~nm})$ with slight desaturation because response of the red color receptor is greater than green .Nanometer techniques are generally used in digital painting, printing etc.

5. Colour components - Light is very much complex in its composition of wavelengths. Colours change with each degree change in wavelength. But the human eye reduces these to three color components in each location in the visual field of eye. The colour components are the basis of primary or basic colours. Some person could not recognize any colour component and some living organisms can recognize three, four or even more colour components.

6. Color constancy - When an artist uses a limited number of colours painting, the eye tends to compensate by seeing any gray or neutral color as the color which is missing from the color board. In a limited number of colours consisting of red, yellow, black, and white, a mixture of yellow and black will appear as a shade of green, a mixture of red and black will appear as a shade of purple and pure gray will appear bluish (ARTISTS may please check these statements) . 7. Nomenclature of colors - Colors are named in several different ways including hue (shades of red, orange, yellow, green, blue, and violet), saturation, brightness, gloss etc. Some color words are derived from the name of an object of that color, such as orange or indigo while others are abstract, like red.

8. Non spectral colours - There are many color perceptions that by definition cannot be pure spectral colors. A mixture of red and violet light from opposite ends of the spectrum and black, gray and white as well as pink, tan and magenta are some common examples of non spectral colours.

9. Basic, primary, secondary and tertiary colors - All languages have six basic colors like black, white, red, green, blue, and yellow and a set of twelve colours; black, gray, white, pink, red, orange, yellow, green, blue, purple, brown, and azure (other than blue in Russian and derived from mineral azurite).

Mostly human color perceptions are generated by a mixture of three colors yellow, blue and red. These primary colours are used in painting, photography and printing. In nature very rarely completely saturated or pure primary colours are available or possible as compared to colour printing.

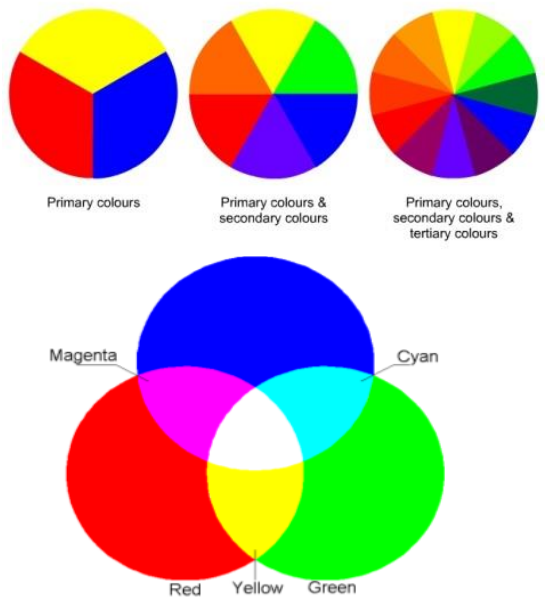

10. Additive and subtractive colors - Blue, green and red are also three primary colours used in television screen, projectors and computer monitors. When combined, these colours produce the appearance of white. By adding different proportions of these three colours, other colours can also be generated.

Yellow, cyan and magenta are called subtractive colours, since each of these colours are produced when one of the primary colour is removed from white light. Subtractive colours are seen when a pigment in an object absorbs a particular colour, while reflects the rest. Subtractive colors also include adding magenta to yellow yields red and adding all three primary colors together yields black. 


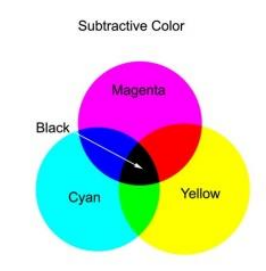

11. Color Contrast - all about the difference - Contrast is the difference in visual properties that makes an object or image more distinguishable from other objects or image and the background. This true with colour contrast also. Colour contrasts are many types in hues, shade, tone, saturation etc.
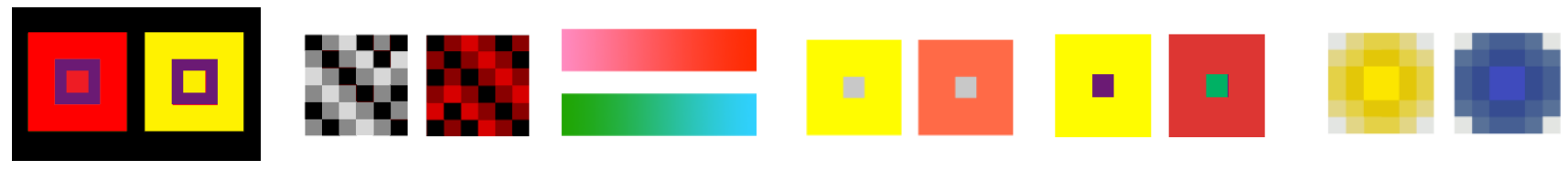

12. Colour surface display - The color surface displays come from the parts of the visible spectrum (light) that are not absorbed by the object and therefore, remain visible. Green leaves look green as they do not absorb green part $(\sim 560-490 \mathrm{~nm})$ of the spectrum and likewise.

14. Universal colour coding - The Munsell's color system is the universal colour coding system and used widely in world by the persons related with scientific study of colours and other objects. ). It was created by Professor Albert H. Munsell in the first decade of the 20th century and adopted by the USDA as the official color system for soil research in the 1930s. Munsell's system has outlasted its contemporary color models, and though it has been superseded for some uses by models.

The Munsell's color system is a color space that specifies colors based on three color coordinates namely Hue, Value and Chroma.

Hue - Hue indicates main colour of the spectrum and main wave length of the light. Yellow, green, blue, purple and red are five principal hues along with 5 intermediate hues (e.g., YR) Values of hues vary from 0 to 10. Increasing numbers hue indicates that colour is becoming more yellow and less red.

Value - Value indicates relative darkness or lightness of the colours. It is relative blackness or whiteness of the colours. On a vertical scale it

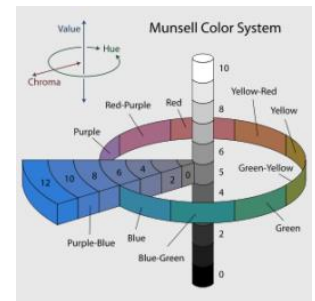
varies from 0 (absolute black) at the bottom to10 (absolute white) at the top. Neutral grays lie along the vertical axis between black and white. Value is approximately equal to square root of total reflected light.

Chroma - Chroma represents the relative purity, strength or saturation of a color. Chroma varies from 0 to 12. Lower chroma shows less purity of the colour i.e., more washed out. A color may be fully specified by the three numbers for hue, value and chroma in that order. $A$ purple colour of medium lightness and fairly saturated would be 5P 5/10 with 5P meaning the color in the middle of the purple hue band, 5/ meaning medium value (lightness) and a chroma of 10 . 


\section{INTERNATIONAL JOURNAL Of RESEARCH -GRANTHAALAYAH \\ A knowledge Repository}

Art

15. False colour composite - Presently available photographic materials (films) are sensitive to a wide range of EMR, i.e. from UV $(230 \mathrm{~nm})$ IR $(900 \mathrm{~nm})$. Visible light is in between these two. Black and white and true colours both in visible range are generally used in common photography.

But UV and IR are mostly used in remote sensing etc. to discriminate among similarly coloured objects. In UV photography various shades of yellow and orange change to green to brown etc. In IR, green colour changes to red to magenta, blue to blue green colour of water to cyan, blue to black colours etc. Similarly contrast between land and water is more distinguishable in IR as compared to visible range of EMR. This helps to identify and study the objects more effectively.

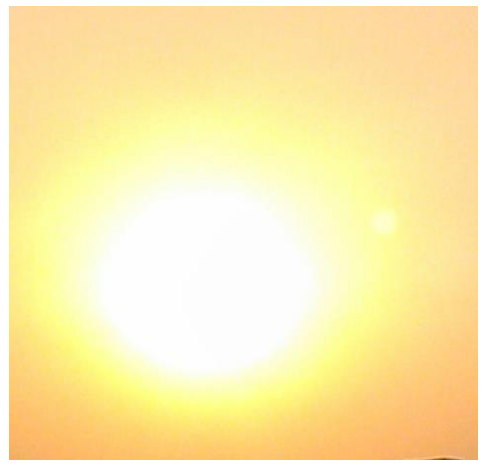

Natural colour image of Sun and sky

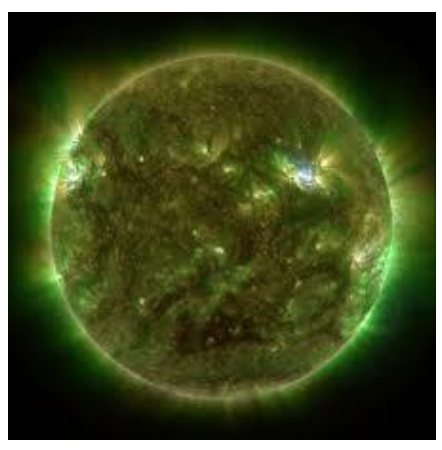

False (UV) colour image of Sun and sky

In Visible light it very difficult to the Sun. But one may see UV imafe of Sun and can identify outer boundry,corona,spots, winds etc. of the Sun.
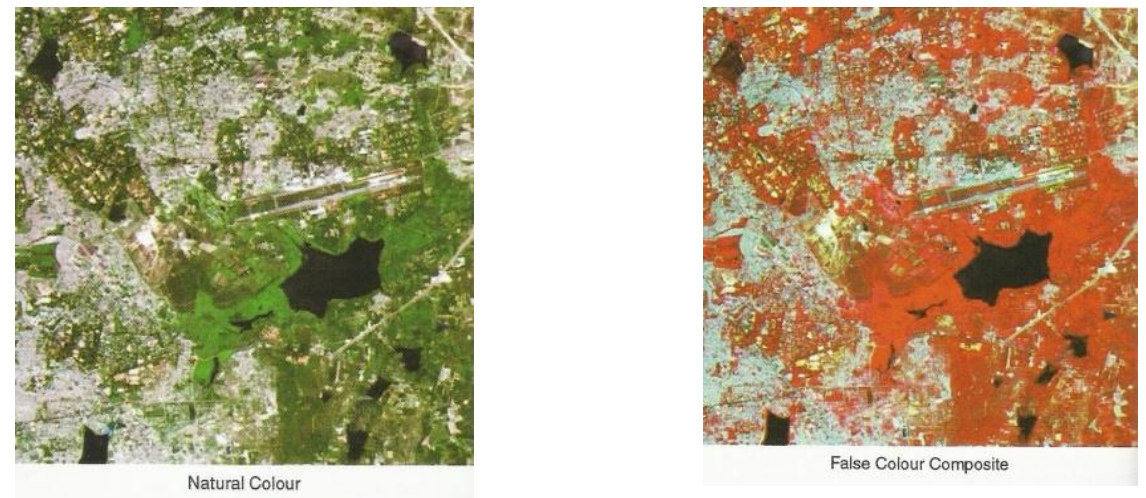

Natural colour is generated using the primary colours- Blue, Green and Red. The green vegetation appears green. The false colour composite image uses Green, Red and Near Infrared bands for blue, green and red colours respectively. Here vegetation appears in different hues of red. 


\section{INTERNATIONAL JOURNAL Of RESEARCH -GRANTHAALAYAH \\ A knowledge Repository}

Art

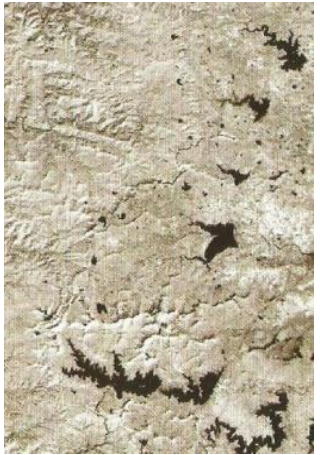

Natural colour image of land and water

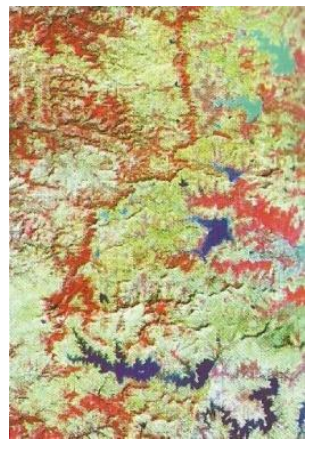

False (NIR) colours image of land and water

In the NIR image all water bodies are seen in dark black tones irrespective of the turbidity levels of the water. There is a very sharp separation between land and water. The FCC image shows water bodies in different shades ranging from dark black to different hues of blue which represent various levels of turbidity, bottom reflection and depth of water body.

\section{REFRENCES:}

1 Johannes Itten, 1970, The Elements of Color, John Wiley \& Sons; 1 edition, ISBN13: $978-0471289296$.

2 George Joseph, 2009, Fundamentals of Remote Sensing, Universities Press, $2^{\text {nd }}$ edition ISBN-13:9788173715358.

3 M.V.K. Sivakumar, 2004, Satellite Remote Sensing and GIS Applications in Agricultural Meteorology, World Meteorological Organization, Geneva, Switzerland.

4 वेंकटेश भारद्वाज, मृदा विज्ञान के मौलिक सिद्धान्त - वैज्ञानिक तथा तकनीकी शब्दावली आयोग, नई दिल्ली तथा प्रकाशन निदेशालय, पन्तनगर, 1997

5 वेंकटेश भारद्वाज तथा अन्य, मृदा विज्ञान परिभाषा कोश - वैज्ञानिक तथा तकनीकी शब्दावली आयोग, नई दिल्ली, 1997.

6 Bharadwaj, Venkatesh - Fundamentals of Soil Science, Laboratory Manual, Pantnagar, 1999.

7 Bharadwaj, Venkatesh- Practical Manual: Salt Affected and other problem soils. Pantnagar, 2002.

8 Bharadwaj, Venkatesh-Climatic change and management of weather for sustainable agriculture.2009 (Agrotech Publishing Academy,Udaipur)

9 Bharadwaj, Venkatesh- Emission of Green Houses Gases from Agriculture Sector and measures for their Mitigation 2009 (Agrotech Publishing Academy,Udaipur ) 